\title{
Alcohol intake and brain structure in a multiethnic elderly cohort
}

\author{
Yian Gu, PhD ${ }^{1}$, Nikolaos Scarmeas, $\mathbf{M D}^{1,2,3,4}$, Erica Eaton Short, MA ${ }^{1}$, José A. Luchsinger, \\ $\mathbf{P h D}^{1,2,5,6}$, Charles DeCarli, MD ${ }^{7,8}$, Yaakov Stern, $\mathbf{P h D}^{1,2,3}$, Jennifer J. Manly, $\mathbf{P h D}^{1,2,3}$, \\ Nicole Schupf, PhD ${ }^{1,2,6}$, Richard Mayeux, MD ${ }^{1,2,3,6}$, and Adam M. Brickman, PhD 1,2,3 \\ ${ }^{1}$ The Taub Institute for Research in Alzheimer's Disease and the Aging Brain, Columbia \\ University, New York, NY \\ ${ }^{2}$ The Gertrude H. Sergievsky Center, Columbia University, New York, NY \\ ${ }^{3}$ The Department of Neurology, Columbia University, New York, NY \\ ${ }^{4}$ National and Kapodistrian University of Athens Medical School, Athens, Greece \\ ${ }^{5}$ The Department of Medicine, Columbia University, New York, NY \\ ${ }^{6}$ The Division of Epidemiology, Joseph P. Mailman School of Public Health, Columbia University, \\ New York, NY \\ ${ }^{7}$ Center for Neuroscience, University of California, Davis, CA \\ ${ }^{8}$ Department of Neurology, University of California, Davis, CA
}

\begin{abstract}
Background \& Aims-Evidence suggests that consuming light-to-moderate amounts of alcohol reduces the risk of dementia and is associated better cognitive function and less cardiovascular disease, relative to those consuming no or heavy alcohol. There are only minimal data on the association between alcohol and brain magnetic resonance imaging (MRI) markers. This study aimed to examine the association between alcohol and brain structure measured with MRI.
\end{abstract}

Methods-In this cross-sectional study, high-resolution structural MRI was collected on 589 multi-ethnic community residents of New York aged $\succeq 65$ with available alcohol intake assessments via a food frequency questionnaire. Total brain volume (TBV), white matter hyperintensity volume (WMHV), and presence of infarcts were derived from MRI scans with

(C) 2013 Elsevier Ltd and European Society for Clinical Nutrition and Metabolism. All rights reserved.

Corresponding author: Yian Gu, PhD, 630 W. $168^{\text {th }}$ Street, New York, NY 10032, Phone: 212-305-6684, Fax: 212-342-1838, yg2121@ columbia.edu.

Statement of authorship: YG contribution to conception and design of the study, carried out the study, data analyses and writing of the manuscript. NS(Scarmeas) contributed to conception and design of the study, carried out data analysis and writing of the manuscript. EES participated in data analysis and writing of the manuscript. CD participated in data collection, data analysis and writing of the manuscript. JAL, CD, YS, JJM, NS(Schupf) and RM provided significant advice and consultation all through the study and participated in writing of the manuscript. AMB contributed to conception and design of the study, and carried out data analysis and writing of the manuscript. All authors read and approved the final manuscript.

Conflicts of interest: The authors report no disclosures.

Publisher's Disclaimer: This is a PDF file of an unedited manuscript that has been accepted for publication. As a service to our customers we are providing this early version of the manuscript. The manuscript will undergo copyediting, typesetting, and review of the resulting proof before it is published in its final citable form. Please note that during the production process errors may be discovered which could affect the content, and all legal disclaimers that apply to the journal pertain. 
established methods. We examined the association of alcohol intake with these imaging markers using regression models adjusted for demographic, clinical, and vascular risk factors.

Results-Compared to non-drinking, light-to-moderate total alcohol $(b=0.007, p=0.04)$ or wine $(b=0.008, p=0.05)$ intake, but not beer or liquor intake, was associated with larger TBV. Further analysis showed a dose-response association between alcohol ( $\mathrm{p}$-trend=0.03) or wine ( $\mathrm{p}$ trend=0.006)) and TBV. Overall, alcohol intake was not associated with WMHV or brain infarcts.

Conclusions-Our study suggests that among older adults in the community, light-to-moderate alcohol intake, in particular wine, is associated with larger TBV. These findings suggest that light to moderate alcohol consumption is potentially beneficial for brain aging, but replication is needed.

\section{Keywords}

Alcohol; nutrition; magnetic resonance imaging (MRI); brain atrophy; cerebrovascular disease; neuroepidemiology

\section{INTRODUCTION}

A large amount of evidence from longitudinal epidemiological studies suggests that people consuming light-to-moderate amounts of alcohol have a lower risk of dementia(1-3) or better cognitive function $(3,4)$, and lower risk of ischemic stroke(5) than do persons who either abstain from or consume heavy amounts of alcohol. It is currently unclear which biological mechanisms could be involved in these associations.

A number of neuroimaging markers, including global and regional brain volume(6), white matter hyperintensity volume (WMHV)(7), and brain infarcts(8), have been related to cognitive functioning, and risk of dementia and stroke. Neuroimaging markers may reflect different biological changes. For instance, decreased relative brain volume represents brain atrophy, and increased WMHV and presence of brain infarcts represent small and larger vessel cerebrovascular disease, respectively. Therefore, examining the associations of alcohol intake with neuroimaging markers may help clarify potential underlying mechanisms for the apparent association between alcohol intake and a protection against neurological or vascular diseases.

The few studies that have examined the association between alcohol consumption and brain MRI markers at a population level had mixed results (9-16). No previous study has examined the effect of alcohol on brain imaging findings among diverse populations that include Hispanics. In addition, different alcoholic beverages may share the same alcohol content, but they also contain different non-alcoholic ingredients such as anti-oxidant polyphenols, which can be found in wine but not in beer or liquor(17). Thus, wine, beer, and liquor may be protective or may confer risk for suboptimal brain aging via different brain markers. However, the independent role of beer, wine, and liquor has rarely been assessed. In an attempt to fill some of these gaps, we sought to examine the association between alcohol (total alcohol, beer, wine, and liquor) intake and brain volume, WMHV, and brain infarcts among participants of the Washington Heights/Hamilton Heights Inwood Columbia Aging Project (WHICAP), a community-based study of multiethnic, elderly (65 years or 
older) people in New York. Based on previous reports of a protective cognitive and vascular effect of moderate alcohol consumption, we hypothesized that light-to-moderate alcohol consumption would be associated with markers of healthier brain aging (1-5).

\section{MATERIALS AND METHODS}

\section{Study Participants}

The WHICAP participants were identified (via ethnicity and age stratification processes) from a probability sample of Medicare beneficiaries aged 65 or older, residing in northern Manhattan(18). The initial sample for this study included 2,776 participants of the ongoing WHICAP II cohort. Briefly, at entry, a physician elicited each participant's medical and neurological history, and conducted a standardized physical and neurological examination. Each participant also underwent a structured in-person interview including an assessment of health and function and a neuropsychological battery(18). Participants were followed at intervals of approximately 1.5 years, repeating the baseline examination and consensus diagnosis. The diagnosis of any type of dementia or its absence was based on standard research criteria, using all available information (except the MRI results) at a consensus conference of physicians, neurologists, neuropsychologists and psychiatrists. The diagnosis was made blind to alcohol consumption information. The Columbia University Institutional Review Board has reviewed and approved this project. All individuals provided written informed consent.

In 2004, we began systematic collection of high-resolution neuroimaging data on ongoing dementia-free WHICAP II participants $(19,20)$. A total of 769 WHICAP participants received MRI scans(20). Fifty-two subjects met diagnostic criteria for dementia at the clinical evaluation closest to neuroimaging were further excluded. Of the remaining 717 subjects, 114 had no alcohol assessments available at the MRI visit. The 603 subjects with alcohol assessments were demographically and clinically similar (data not shown) to those 114 subjects with missing alcohol assessment.

\section{MRI protocol}

Scan acquisition was performed on a 1.5T Philips Intera scanner at Columbia University Medical Center and transferred electronically to the University of California at Davis for morphometric analysis(20).

User-operated image analysis was performed on a workstation (Ultra 5; Sun Microsystems, Santa Clara, California) using the Quantium 6.2 software package (Sun Microsystems). Subject identifying information was not available to the operator. We have previously reported the methods used to derive the two morphologic variables, i.e., total brain volume (TBV) and WMHV(20). TBV was the sum of voxels designated as brain volume from the segmentation process. WMHV was calculated as the sum of voxels $3.5 \mathrm{SD}$ or greater above the mean intensity value of the image and multiplied by voxel dimensions and section thickness. TBV was adjusted for total cranial volume (TCV), and the ratio of TBV to TCV [i.e. relative TBV $(\mathrm{rTBV})=\mathrm{TBV} / \mathrm{TCV} \times 100$ ] was used in the analyses. Similarly, WMHV was adjusted for TCV, and relative WMHV (rWMHV) was used in the analyses. 
The presence or absence of brain infarction on MRI was determined using all available images, as previously described(19). Only lesions $23 \mathrm{~mm}$ qualified for consideration as brain infarcts. Two raters determined the presence of cerebral infarction on MRI. Previously published $\kappa$ values for agreement among raters have been generally good, ranging from 0.73 to $0.90(19)$.

\section{Alcohol consumption}

Information about average alcohol consumption (beer, wine, or liquor) over the prior year was obtained using the 61-item version of Willett's semi-quantitative food frequency questionnaire (SFFQ) (Channing Laboratory, Cambridge, MA), administered by trained interviewers in English or Spanish. Participants reported one of the following combinations of frequency and amount of alcohol consumption: <1 drink/month, 1-3 drinks/month, 1 drink/week, 2-4 drinks/week, 5-6 drinks/week, one drink/day, 2-3 drinks/day, 4-5 drinks/ day, and $\geq 6$ drinks/day(1). One drink was 12 -oz beer, 4-oz wine, or 1.5-oz liquor, each containing approximately $10 \mathrm{~g}$ of alcohol. These serving frequencies were converted to monthly consumption of alcohol drinks. Because of the U-shape relationship between alcohol intake and the neurological outcomes(1-5) and the small number of heavy drinkers in this study population, participants were classified into two categories: none (currently not drinking), light-to-moderate ( $>0$ and $\leq 30$ drinks/month for women and $>0$ and $\leq 60$ drinks/ month for men), and heavy (>30 and $>60$ drinks/month for women and men, respectively) drinkers according to the Dietary Guidelines for Americans 2010 definition of moderate drinking (up to 1 drink per day for women and up to 2 drinks per day for men) (21). A total of 409, 180 and 14 subjects reported no, light-to-moderate, and heavy drinking of alcohol, respectively. Considering the small number of heavy-drinkers, and the potential difference between non-drinkers and heavy-drinkers, we included in the analysis only the 589 nondrinkers and light-to-moderate drinkers. The measurements of alcohol intake between two SFFQs administered 2 months apart were not significantly different, suggesting a satisfying reliability of alcohol measurement by SFFQ in the current study population(1).

\section{Covariates}

Age (years), education (years), caloric intake (kcal), and body mass index (BMI; weight in kilograms divided by height in square meters $\left[\mathrm{kg} / \mathrm{m}^{2}\right]$ ) were used as continuous variables. Participants were assigned to one of four groups: African American (Black non-Hispanic), Hispanic, White (non-Hispanic) or Other based on self-report using the format of the 1990 US census. Ethnicity was used as a dummy variable with non-Hispanic White and Other as the reference. Sex was used as a dichotomous variable with male as the reference.

Apolipoprotein (APOE) genotype was used dichotomously: absence of $\varepsilon 4$ allele (as reference) vs. presence of either 1 or $2 \varepsilon 4$ alleles. Smoking status was used dichotomously: ever smoke vs. never smoked, with never smoked as the reference group. History of diabetes, hypertension, heart disease, or clinical stroke was ascertained by self-report from an interview with participants and/or their informants and was each used as a dichotomous variable with no history as the reference. 


\section{Statistical analyses}

Characteristics of participants by alcohol consumption category were compared using t-test for continuous variables and $\chi^{2}$ test for categorical variables. The distribution of rWMHV was positively-skewed; therefore, we used a natural log transformed rWMHV in the analysis. Logistic regression models with presence or absence of one or more brain infarct as the outcome, or linear regression models with rWMHV or rTBV as the outcome, were used to assess the association between alcohol consumption and brain MRI markers, firstly unadjusted, and then adjusted for age, gender, ethnicity, education, ever-smoke, caloric intake, and APOE genotype (basic adjusted model), and then further adjusted for BMI and four vascular disease history variables (diabetes, hypertension, and heart disease) (fully adjusted model). We initially included total alcohol intake as the main predictor variable in the models. To examine whether the association between alcohol and MRI markers was driven by any particular beverage type, we included the intakes of three types of beverages (i.e. beer, wine or liquor) simultaneously in the models. Potential effect modification by sex, APOE genotype, education, or ethnicity was examined by including the interaction terms in basic adjusted regression models. If significant interactions were found, stratified analysis based on the effect modifier would be performed.

Supplementary analysis-As previous studies have shown that alcohol intake contributed to brain atrophy in a dose-response fashion $(12,13,15)$, we performed a supplementary analysis to explore whether there is a dose-response association between alcohol and MRI markers across the alcohol drinking levels. All the light-to-moderate drinkers were further categorized into two groups: light drinkers ( $<8$ drinks per month), and moderate drinkers ( $\geq 8$ drinks per month but less than heavy drinking) for both men and women.

As ex-heavy drinkers might have ceased alcohol use due to health issues and assigning them in the non-drinker group might bias the results, we performed a supplementary analysis by excluding past-alcoholic participants and past heavy-drinkers. Past-alcoholism information was collected as part of the medical history assessment for the WHICAP participants, and past heavy-drinkers were identified by examining the past SFFQs using the same definition as described earlier.

It has been reported that alcohol consumption decreases following clinical diagnosis of stroke(22). Thus, to increase our confidence that the findings are not reflecting a reversed association, we examined the association of alcohol consumption with MRI-measured silent brain infarcts by excluding 68 subjects with clinical stroke.

We also performed analysis of each of the three types of beverage without controlling for the other two, i.e., only one type of beverage in the regression models.

All analyses were conducted using PASW Statistics (IBM, Chicago, IL, USA). All p-values were based on two-sided tests. The significance level was set at 0.05 for all tests. 


\section{RESULTS}

\section{Clinical-demographic-MRI markers characteristics and alcohol consumption}

The mean (SD) age of the sample at time of MRI scan was 80.1 (5.5), and $67 \%$ were women. A total of $180(31 \%), 82(14 \%), 121$ (21\%), and 77 (13\%) of all participants reported light-to-moderate total alcohol, beer, wine, and liquor intakes, respectively.

Compared to non-drinkers, those with light-to-moderate total alcohol intake were less likely to be female, had more years of education, were more likely to be White and less likely to be Hispanic, were more likely to have smoked, were less likely to have diabetes history, had higher daily caloric intake, and had lower BMI (Table 1).

Compared to non-drinkers, those with light-to-moderate beer, wine, or liquor intake had more years of education, and were more likely to be White or Black and less likely to be Hispanic; those who drank beer or liquor to a moderate degree were less likely to be female but more likely to ever-smokers; those with light-to-moderate wine or liquor intake were less likely to have diabetes history; those with light-to-moderate beer intake were less likely to have hypertension history; those with light-to-moderate wine intake had lower BMI; and beer consumers tended to have higher daily caloric intake (eTable 1).

\section{Alcohol consumption and brain MRI markers}

Compared to non-drinkers, participants with light-to-moderate total alcohol intake had larger rTBV (i.e., less brain atrophy) in basic adjusted models (Table 2). This association was attenuated in the fully adjusted model (Table 2). Total alcohol intake was not associated with rWMHV, or the odds of brain MRI infarcts (Table 2).

When beer, wine, and liquor intakes were included simultaneously in the models, participants with light-to-moderate wine intake had larger rTBV compared with non-winedrinkers in the basic adjusted model (Table 2) although it was also attenuated in the fully adjusted model $(\beta=0.007, \mathrm{p}=0.09)$. Wine intake was not associated with other MRI markers. Beer or liquor intake was not associated with any of the MRI markers.

Sex, ethnicity, education, or APOE genotype did not modify the association between alcohol intake and brain MRI markers (data not shown). We found a significant interaction between education and beer consumption for rWMHV ( $\mathrm{p}$ for interaction $=0.005$ ). We then ran stratified analysis based on education higher or lower than the median level (12 years). Stratified analysis showed that beer intake was associated with larger WMHV in lower education subjects but not in higher education subjects. No significant interaction was found for education and alcohol, wine, or liquor on any of the brain MRI markers.

\section{Supplementary Analyses}

When light and moderate alcohol drinkers were separately compared to non-drinkers, no difference was found between the rTBV in light drinkers and that in non-drinkers, but the rTBV in moderate drinkers was significantly larger than that in non-drinkers in the basic adjusted model $(\beta=0.009, \mathrm{p}=0.05$; eTable 2$)$. Furthermore, there is a significant trend ( $\mathrm{p}$ trend $=0.03$ ) indicating that increase in total alcohol consumption was associated with 
increased brain volume. Similar to the findings in main analysis, a significant association with rTBV was found only for wine (p-trend=0.006) but not for beer or liquor consumption (eTable 2).

Excluding 12 past-alcoholic participants and 17 past-heavy drinkers in general did not change the results much: light-to-moderate total alcohol drinking was still significantly associated with larger rTBV $(\beta=0.007, p=0.04)$ and in a dose-response fashion ( $p$ for trend $=0.02$ ) in the basic adjusted model. Although there was no significant difference in rTBV comparing light-to-moderate wine drinkers with non-drinkers $(b=0.007, p=0.10)$, there was a dose-response association ( $\mathrm{p}$ for trend $=0.01$ ) between wine consumption and rTBV, with $\beta=0.02(\mathrm{p}=0.009)$ and $0.004(\mathrm{p}=0.40)$ for moderate- and light-drinkers compared with nondrinkers, respectively. There was no association between beer or liquor with rTBV, and alcohol (total or any type) drinking was not associated with either rWMHV or odds of brain infarcts (data not shown).

When beer, wine, or liquor was analyzed without adjusting for the other two types of beverage, the results were essentially unchanged from the models simultaneously including three types of beverage (data not shown).

Unsurprisingly, participants with presence of MRI brain infarcts were more likely to report a history of clinical stroke than subjects absent of brain infarcts ( $22.3 \%$ vs. $6.2 \%, \mathrm{p}<0.0001)$. Excluding subjects with clinical stroke $(\mathrm{n}=68)$ did not notably change the results. In the basic adjusted model, compared to non-drinkers, Odds Ratio (OR) of MRI infarcts was 1.05 (95\%CI: 0.67-1.65; $\mathrm{p}=0.82$ ) for light-to-moderate alcohol drinkers.

\section{DISCUSSION}

In this cross-sectional study of an elderly population without dementia, we found participants consumed a light-to-moderate amount of total alcohol had larger relative brain volumes than non-drinkers, and this association was likely to be driven by wine consumption. No significant association between alcohol consumption and WMHV or brain infarcts risk was observed in the current study.

Our findings on brain atrophy were consistent with several previous studies. In an Australian study, higher alcohol consumption was related with increased gray matter in men(9). In another study, lifetime alcohol intake was associated with higher white-matter density in the right frontal gyrus and the right parietal region(10). Another study found alcohol intake was positively associated with hippocampus and amygdala volumes in APOE $\varepsilon 4$ carriers(11). Nevertheless, some other previous studies have found higher alcohol consumption was associated with more brain atrophy(12-16). Given the inconsistency of the literature, further studies are warranted.

The mechanism for the potential beneficial effect of light-to-moderate alcohol intake on TBV is unknown, but it might be due to alcohol's functions on reduction of inflammation; reduction of low-density lipoprotein and increase of high-density lipoprotein; reduction of insulin resistance and increase in insulin sensitivity(23). In addition, light-to-moderate alcohol intake has been consistently related with lower risk of cardiovascular diseases(23), 
which in turn has been related with less brain atrophy(24). In the current study, we also found that light-to-moderate alcohol drinkers reported less diabetes history, and participants with no diabetes history had larger TBV (less atrophy) compared to those with diabetes history. Indeed, the association between alcohol intake and brain atrophy was attenuated when adjusted for diabetes (along with other vascular factors), suggesting that vascular factors might mediate the association between alcohol and brain atrophy.

Further analysis in the current study also showed that this association might be driven by wine consumption, but not other types of alcoholic beverages. This is in line with a previous report of the WHICAP population(1), which showed that light-to-moderate intake of wine, but not beer or liquor, was associated with reduced risk of AD in APOE $\varepsilon 4$ non-carriers. The neuroprotective effects of wine may be partly attributed to the alcohol. However, our findings that only wine but not beer or liquor was associated with brain volume indicated that the protective properties of red wine may more likely to be related with the flavonoids and polyphenols (especially resveratrol), which are abundant in wine (particularly red wine) but present only at a very low amounts in beers or other alcohol beverages(17). Flavonoids and polyphenols have been reported to exert numerous beneficial health effects including anti-inflammatory(25) anti-oxidative(26), and beneficial vascular effects(27).

The evidence on the relationship between alcohol consumption on markers of cerebrovascular disease, i.e. WMHV and brain infarcts, is inconsistent. A Japanese study also found light and moderate alcohol drinkers had larger WMHV and increased risk of infarcts than non-drinkers(15). In contrast, in Cardiovascular Health Study, moderate alcohol drinkers had a lower prevalence of white matter abnormalities compared to nondrinkers or heavy drinkers(12). Investigators from Rotterdam also found moderate drinking was associated with less WMHV, but not associated with infarcts(11). Similar to other studies $(9,13,14)$, we did not find an association between alcohol consumption and WMHV or infarcts in the overall sample. Increasing age is known to be associated with higher WMHV(28). Thus, the effect alcohol has on MRI markers that is above and beyond the aging effect may become increasingly difficult to detect in our study population with an average age of 80, as aging may play a dominant role in brain MRI markers in an older population. However, we did find a significant association between higher beer intake and larger WMHV among lower education participants. It is possible that higher educated subjects might have some healthier lifestyles which could mitigate the potential effect of beer on WMHV. However, it remained to be confirmed whether the beer-WMHV association exists only among subjects with relatively low education.

Overall, the current evidence on alcohol and MRI markers is inconsistent. The discrepancy among the studies can be explained by many factors including study populations' demographic characteristics such as age and ethnicity, comorbidities, alcohol consumption assessment, MRI procedures, or other confounding factors. Different ages represented across studies suggest differential lifetime cumulative alcohol consumption, which could be more relevant to brain changes. Studies also differ in the definition of "moderate alcohol drinking”. For example, while a majority of studies(12-14) considered " $\geq 15$ drinks per week" as "heavy drinking", one study considered only those consuming “ $\geq 28$ drinks per 
week" as heavy drinkers(11). Finally, not all studies excluded participants with clinical dementia or stroke, and the comorbidity burden due to other diseases has been different.

Our study has many strengths. While most of the previous studies examining alcohol and MRI markers included predominantly a single race/ethnicity group, either of European origin or of Asian origin, our study included a multiethnic group of participants and is among the first to include Hispanic participants. Our study population is also diverse in other demographic measures. Thus, the findings of the current study are likely to reflect the general population in the US, which is becoming increasingly diverse(20). We excluded participants with dementia, limiting the possibility that the recall of alcohol intake is affected by memory deficits. Measures for multiple potential confounding factors have been carefully recorded and adjusted for in the analyses. In addition to total alcohol intake, we also examined the individual roles of beer, wine, and liquor on brain imaging markers.

Some limitations of the current study need to be noted. Given that our study is crosssectional, we cannot infer causality of the associations. Subjects may change their alcohol consumption habits over time so that ex-heavy drinkers might have ceased drinking due to health problems. Nevertheless, excluding past-alcoholic and past-heavy-drinking subjects did not notably change our results. The actual drinking habit and amount of the participants may be underestimated using self-reported SFFQ; nevertheless underestimation is mostly likely to occur in abuse rather than moderate alcohol use(29). We assessed average alcohol intake over a one year period prior to MRI scan, but it would also be interesting to examine lifetime cumulative and middle-life alcohol intakes. Although we controlled for a wide variety of potential confounders, we cannot rule out the possibility of residual confounding. For instance, socioeconomic status may not be fully explained by education, and other factors such as household income were not considered in the analysis and might have confounded the results. We did not control for genotypes of the gene encoding acetaldehyde dehydrogenase, an enzyme involved in alcohol metabolism(30).

Our study suggests that moderate alcohol intake might be associated with larger brain volumes in elderly people. The potential benefits of moderate wine consumption on brain volume need to be confirmed in future studies, especially longitudinal ones.

\title{
Supplementary Material
}

Refer to Web version on PubMed Central for supplementary material.

\section{Acknowledgments}

This work is supported by NIH grants AG07232, AG028506, AG029949, and K99AG042483.

\section{Non-standard abbreviations}

\author{
APOE Apolipoprotein \\ MRI magnetic resonance imaging \\ SFFQ semi-quantitative food frequency questionnaire
}




$\begin{array}{ll}\text { TBV } & \text { total brain volume } \\ \text { TCV } & \text { total cranial volume } \\ \text { WHICAP } & \text { Washington Heights/Hamilton Heights Inwood Columbia Aging Project } \\ \text { WMHV } & \text { white matter hyperintensity volume }\end{array}$

\section{References}

1. Luchsinger JA, Tang MX, Siddiqui M, Shea S, Mayeux R. Alcohol intake and risk of dementia. J Am Geriatr Soc. 2004; 52:540-546. [PubMed: 15066068]

2. Ruitenberg A, van Swieten JC, Witteman JC, Mehta KM, van Duijn CM, Hofman A, et al. Alcohol consumption and risk of dementia: the Rotterdam Study. Lancet. 2002; 359:281-286. [PubMed: 11830193]

3. Mukamal KJ, Kuller LH, Fitzpatrick AL, Longstreth WT Jr, Mittleman MA, Siscovick DS. Prospective study of alcohol consumption and risk of dementia in older adults. JAMA. 2003; 289:1405-1413. [PubMed: 12636463]

4. Stampfer MJ, Kang JH, Chen J, Cherry R, Grodstein F. Effects of Moderate Alcohol Consumption on Cognitive Function in Women. N Engl J Med. 2005; 352:245-253. [PubMed: 15659724]

5. Reynolds K, Lewis LB, Nolen JDL, Kinney GL, Sathya B, He J. Alcohol Consumption and Risk of Stroke: A Meta-analysis. Journal of the American Medical Association. 2003; 289:579-588. [PubMed: 12578491]

6. Resnick SM, Pham DL, Kraut MA, Zonderman AB, Davatzikos C. Longitudinal magnetic resonance imaging studies of older adults: A shrinking brain. J Neurosci. 2003; 23:3295-3301. [PubMed: 12716936]

7. Brickman AM, Honig LS, Scarmeas N, Tatarina O, Sanders L, Albert MS, et al. Measuring cerebral atrophy and white matter hyperintensity burden to predict the rate of cognitive decline in Alzheimer disease. Arch Neurol. 2008; 65:1202-1208. [PubMed: 18779424]

8. Vermeer SE, Prins ND, den Heijer T, Hofman A, Koudstaal PJ, Breteler MM. Silent brain infarcts and the risk of dementia and cognitive decline. N Engl J Med. 2003; 348:1215-1222. [PubMed: 12660385]

9. Anstey KJ, Jorm AF, Reglade-Meslin C, Maller J, Kumar R, von Sanden C, et al. Weekly alcohol consumption, brain atrophy, and white matter hyperintensities in a community-based sample aged 60 to 64 years. Psychosom Med. 2006; 68:778-785. [PubMed: 17012533]

10. de Bruin EA, Hulshoff Pol HE, Schnack HG, Janssen J, Bijl S, Evans AC, et al. Focal brain matter differences associated with lifetime alcohol intake and visual attention in male but not in female non-alcohol- dependent drinkers. Neuroimage. 2005; 26:536-545. [PubMed: 15907310]

11. den Heijer T, Vermeer SE, van Dijk EJ, Prins ND, Koudstaal PJ, van Duijn CM, et al. Alcohol intake in relation to brain magnetic resonance imaging findings in older persons without dementia. Am J Clin Nutr. 2004; 80:992-997. [PubMed: 15447910]

12. Mukamal KJ, Longstreth WT Jr, Mittleman MA, Crum RM, Siscovick DS. Alcohol consumption and subclinical findings on magnetic resonance imaging of the brain in older adults: the cardiovascular health study. Stroke. 2001; 32:1939-1946. [PubMed: 11546878]

13. Ding J, Eigenbrodt ML, Mosley TH Jr, Hutchinson RG, Folsom AR, Harris TB, et al. Alcohol intake and cerebral abnormalities on magnetic resonance imaging in a community-based population of middle-aged adults: the Atherosclerosis Risk in Communities (ARIC) study. Stroke. 2004; 35:16-21. [PubMed: 14657449]

14. Paul CA, Au R, Fredman L, Massaro JM, Seshadri S, Decarli C, et al. Association of alcohol consumption with brain volume in the Framingham study. Arch Neurol. 2008; 65:1363-1367. [PubMed: 18852353] 
15. Fukuda K, Yuzuriha T, Kinukawa N, Murakawa R, Takashima Y, Uchino A, et al. Alcohol intake and quantitative MRI findings among community dwelling Japanese subjects. J Neurol Sci. 2009; 278:30-34. [PubMed: 19059611]

16. Taki Y, Kinomura S, Sato K, Goto R, Inoue K, Okada K, et al. Both global gray matter volume and regional gray matter volume negatively correlate with lifetime alcohol intake in non-alcoholdependent Japanese men: a volumetric analysis and a voxel-based morphometry. Alcohol Clin Exp Res. 2006; 30:1045-1050. [PubMed: 16737464]

17. Pinder RM, Sandler M. Alcohol, wine and mental health: Focus on dementia and stroke. J Psychopharmacol (Oxf). 2004; 18:449-456.

18. Stern Y, Andrews H, Pittman J, Sano M, Tatemichi T, Lantigua R, et al. Diagnosis of dementia in a heterogeneous population. Development of a neuropsychological paradigm-based diagnosis of dementia and quantified correction for the effects of education. Arch Neurol. 1992; 49:453-460. [PubMed: 1580806]

19. Scarmeas N, Luchsinger JA, Stern Y, Gu Y, He J, DeCarli C, et al. Mediterranean diet and magnetic resonance imaging-assessed cerebrovascular disease. Ann Neurol. 2011; 69:257-268. [PubMed: 21387371]

20. Brickman AM, Schupf N, Manly JJ, Luchsinger JA, Andrews H, Tang MX, et al. Brain morphology in older African Americans, Caribbean Hispanics, and whites from northern Manhattan. Arch Neurol. 2008; 65:1053-1061. [PubMed: 18695055]

21. U.S. Department of Agriculture and U.S. Department of Health and Human Services. Dietary Guidelines for Americans, 2010. 7. Washington, DC: U.S. Government Printing Office; Dec. 2010

22. Newsom JT, Huguet N, McCarthy MJ, Ramage-Morin P, Kaplan MS, Bernier J, et al. Health behavior change following chronic illness in middle and later life. J Gerontol B Psychol Sci Soc Sci. 2011; 67:279-288. [PubMed: 21983040]

23. Collins MA, Neafsey EJ, Mukamal KJ, Gray MO, Parks DA, Das DK, et al. Alcohol in moderation, cardioprotection, and neuroprotection: epidemiological considerations and mechanistic studies. Alcohol Clin Exp Res. 2009; 33:206-219. [PubMed: 19032583]

24. Schmidt R, Launer LJ, Nilsson LG, Pajak A, Sans S, Berger K, et al. Magnetic resonance imaging of the brain in diabetes: the Cardiovascular Determinants of Dementia (CASCADE) Study. Diabetes. 2004; 53:687-692. [PubMed: 14988253]

25. Zhang F, Liu J, Shi JS. Anti-inflammatory activities of resveratrol in the brain: role of resveratrol in microglial activation. Eur J Pharmacol. 2010; 636:1-7. [PubMed: 20361959]

26. Nigdikar SV, Williams NR, Griffin BA, Howard AN. Consumption of red wine polyphenols reduces the susceptibility of low-density lipoproteins to oxidation in vivo. Am J Clin Nutr. 1998; 68:258-265. [PubMed: 9701181]

27. Oomen CA, Farkas E, Roman V, van der Beek EM, Luiten PG, Meerlo P. Resveratrol preserves cerebrovascular density and cognitive function in aging mice. Front Aging Neurosci. 2009; 1:4. [PubMed: 20552055]

28. Longstreth WT Jr, Arnold AM, Manolio TA, Burke GL, Bryan N, Jungreis CA, et al. Clinical correlates of ventricular and sulcal size on cranial magnetic resonance imaging of 3,301 elderly people. The Cardiovascular Health Study. Collaborative Research Group. Neuroepidemiology. 2000; 19:30-42. [PubMed: 10654286]

29. Stockwell T, Donath S, Cooper-Stanbury M, Chikritzhs T, Catalano P, Mateo C. Under-reporting of alcohol consumption in household surveys: a comparison of quantity-frequency, graduatedfrequency and recent recall. Addiction. 2004; 99:1024-1033. [PubMed: 15265099]

30. Quertemont E, Grant KA, Correa M, Arizzi MN, Salamone JD, Tambour S, et al. The role of acetaldehyde in the central effects of ethanol. Alcoholism: Clinical and Experimental Research. $2005 ; 29: 221-234$. 


\section{Table 1}

Participants' characteristics according to categories of total alcohol intake.

\begin{tabular}{|c|c|c|c|c|}
\hline \multirow[b]{2}{*}{ Characteristics } & \multirow[b]{2}{*}{ Total } & \multicolumn{3}{|c|}{ Total alcohol intake } \\
\hline & & None & Light-to-Moderate & $\mathbf{p}$ \\
\hline Number of participants & 589 & 409 & 180 & \\
\hline Age at scan, mean $(\mathrm{SD})$, years & $80.1(5.5)$ & $80.4(5.6)$ & $79.5(5.4)$ & 0.07 \\
\hline Female, $\mathrm{N}(\%)$ & $395(67)$ & $291(71)$ & $104(58)$ & 0.001 \\
\hline Education, mean(SD), years & $10.8(4.7)$ & $10.0(4.8)$ & $12.5(4.1)$ & $<0.0001$ \\
\hline White, $\mathrm{N}(\%)$ & $157(28)$ & $91(23)$ & $66(40)$ & \\
\hline Black, $\mathrm{N}(\%)$ & $193(35)$ & $134(34)$ & $59(35)$ & 0 \\
\hline Hispanic, $\mathrm{N}(\%)$ & $191(34)$ & $154(40)$ & $37(22)$ & $<0.0001$ \\
\hline Other race, $\mathrm{N}(\%)$ & $15(3)$ & $10(3)$ & $5(3)$ & \\
\hline $\operatorname{APOE}(\varepsilon 4+), \mathrm{N}(\%)$ & $145(29)$ & $94(24)$ & $51(30)$ & 0.17 \\
\hline Ever-smoke, $\mathrm{N}(\%)$ & $315(54)$ & $202(49)$ & $113(63)$ & 0.003 \\
\hline Caloric intake, mean(SD), kcal & $1394(536)$ & $1358(518)$ & $1476(547)$ & 0.01 \\
\hline Diabetes, $\mathrm{N}(\%)$ & $128(22)$ & $100(24)$ & $28(16)$ & 0.02 \\
\hline Heart Disease, $\mathrm{N}(\%)$ & $128(22)$ & $89(22)$ & $39(22)$ & 0.98 \\
\hline Hypertension, $\mathrm{N}(\%)$ & $394(67)$ & $283(69)$ & $111(62)$ & 0.07 \\
\hline Stroke, N(\%) & $68(11)$ & $48(12)$ & $20(11)$ & 0.83 \\
\hline BMI, mean(SD), $\mathrm{kg} / \mathrm{m}^{2}$ & $27.6(5.5)$ & $28.1(5.7)$ & $26.6(5.0)$ & 0.004 \\
\hline rWMHV, mean(SD) & $0.012(0.01)$ & $0.012(0.01)$ & $0.013(0.01)$ & 0.55 \\
\hline rTBV, mean(SD) & $0.728(0.04)$ & $0.727(0.04)$ & $0.730(0.04)$ & 0.51 \\
\hline Presence of infarcts, $\mathrm{N}(\%)$ & $184(32)$ & $122(30)$ & $62(35)$ & 0.24 \\
\hline
\end{tabular}




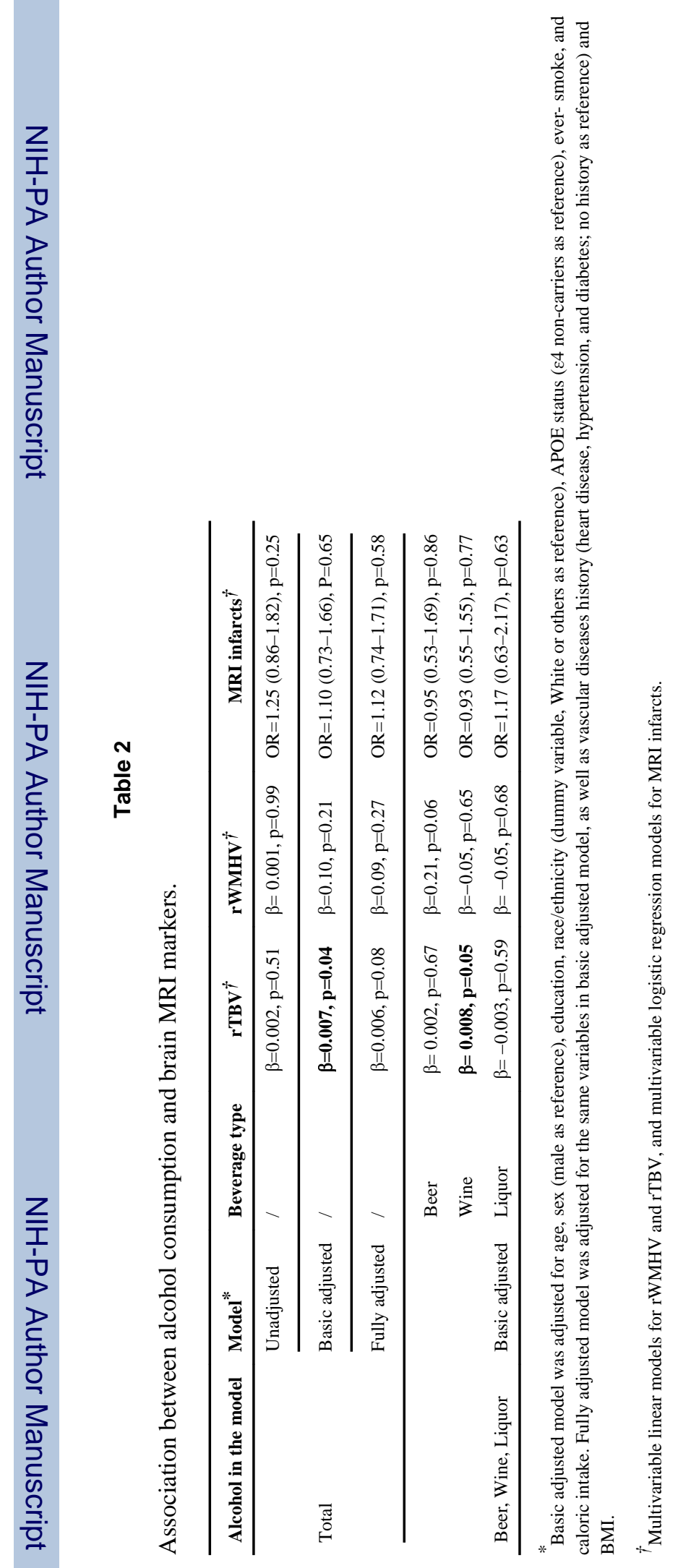

Clin Nutr. Author manuscript; available in PMC 2015 August 01. 